\title{
Convergence Theorems for $m$-Coordinatewise Negatively Associated Random Vectors in Hilbert Spaces
}

\author{
Lyurong Shi \\ Department of Foundational Teaching, Wuhu Institute of Technology, Wuhu 241003, China \\ Correspondence should be addressed to Lyurong Shi; slr@whit.edu.cn
}

Received 7 May 2021; Revised 2 June 2021; Accepted 14 June 2021; Published 20 July 2021

Academic Editor: Xiaodi Li

Copyright $\odot 2021$ Lyurong Shi. This is an open access article distributed under the Creative Commons Attribution License, which permits unrestricted use, distribution, and reproduction in any medium, provided the original work is properly cited.

In this study, some new results on convergence properties for $m$-coordinatewise negatively associated random vectors in Hilbert space are investigated. The weak law of large numbers, strong law of large numbers, complete convergence, and complete moment convergence for linear process of $H$-valued $m$-coordinatewise negatively associated random vectors with random coefficients are established. These results improve and generalise some corresponding ones in the literature.

\section{Introduction}

The random variables $X_{1}, X_{2}, \ldots, X_{n}$ are said to be negatively associated (NA, in short) if, for every pair of disjoint subsets $A$ and $B$ of $\{1,2, \ldots, n\}$ and any real coordinatewise nondecreasing (or nonincreasing) functions $f_{1}$ on $\mathbb{R}^{|A|}$ and $f_{2}$ on $\mathbb{R}^{|B|}$,

$$
\operatorname{Cov}\left(f_{1}\left(X_{i}, i \in A\right), f_{2}\left(X_{j}, j \in B\right)\right) \leq 0,
$$

whenever the covariance above exists, where $|A|$ and $|B|$ denote the cardinalities of $A$ and $B$, respectively. A sequence $\left\{X_{i}, i \geq 1\right\}$ of random variables is NA if every finite subcollection is NA.

The concept of NA random variables can be seen in JoagDev and Proschan [1], which also illustrated that many wellknown multivariate distributions, such as multinomial, convolution of unlike multinomial, multivariate hypergeometric, permutation distribution, negatively correlated normal distribution, and joint distribution of ranks, all satisfy the NA property.

Because of its wide applications, the concept of NA random variables was extended to many different directions. For example, Chandra and Ghosal [2] extended NA to asymptotically almost negative association (AANA); Hu et al.
[3] extended the concept of NA random variables to $m$-NA random variables; Zhang and Wang [4] generalised it to a more broad case, i.e., asymptotically negative association (ANA); Zhang [5] extended it to $\mathbb{R}^{d}$-valued random vectors; Ko et al. [6] introduced the concept of NA random vectors taking values in real separable Hilbert spaces.

Since the concept of NA random vectors was introduced, some related works were established. For the details, we refer to Miao [7], for the Hajeck-Renyi inequality, Thanh [8], for the almost sure convergence, and Hien and Thanh [9], for weak laws of large numbers, among others. For more recent studies, we refer to $\mathrm{Hu}$ et al. [10], Li et al. [11], Yang et al. [12], Yang et al. [13], Fang et al. [14, 15], Mei et al. [16], and Yuan et al. [17].

Let $H$ be a real separable Hilbert space with the norm $\|\cdot\|$ generated by an inner product $\langle\cdot, \cdot\rangle$. Denote $X^{(j)}=\left\langle X, e_{j}\right\rangle$, where $\left\{e^{(j)}, j \in \mathscr{B}\right\}$ is an orthonormal basis in $H, X$ is also a random vector taking values in $H$, and $\mathscr{B}$ is a subset of $\{1,2, \ldots\}$.

Huan et al. [18] first introduced the concept of coordinatewise negatively associated (CNA) random vectors in Hilbert spaces. Huan et al. [18] exemplified that a sequence of NA random vectors is also CNA in Hilbert spaces while the reverse is not true in general. There are also some interesting results concerning the CNA random vectors in 
Hilbert spaces, most of which mainly extended the following Baum-Katz type convergence theorem from independent and identically distributed (i.i.d.) random variables in classical probability space to CNA random vectors in Hilbert space.

Baum-Katz theorem (see [19]): let $p>1, \alpha>(1 / 2)$ and $\alpha p>1$. Let $\left\{X_{n}, n \geq 1\right\}$ be a sequence of i.i.d. random variables with zero mean. Then, the following statements are equivalent:

$$
\begin{aligned}
& \text { (i) } E\left|X_{1}\right|^{p}<\infty \\
& \text { (ii) } \sum_{n=1}^{\infty} n^{\alpha p-2} P\left(\left|\sum_{k=1}^{n} X_{k}\right|>\varepsilon n^{\alpha}\right)<\infty \\
& \text { (iii) } \sum_{n=1}^{\infty} n^{\alpha p-2} P\left(\sup _{k \geq n} k^{-\alpha}\left|\sum_{i=1}^{k} X_{i}\right|>\varepsilon n^{\alpha}\right)<\infty
\end{aligned}
$$

Huan et al. [18] proved the sufficient conditions of the Baum-Katz type complete convergence with $1 \leq p<2$ and $\alpha p>1$, which partially extends Theorem A to CNA random vectors in Hilbert space. Huan [20] considered the case $\alpha p=$ 1 with $1<p<2$. Ko [21] extended the result of Huan et al. [18] to the complete moment convergence, where, however, the case $p=1$ is wrongly proved as pointed out by Huang and $\mathrm{Wu}$ [22]. Ko [23] also established the complete moment convergence with $\alpha p=1$ and $1 \leq p<2$. However, there are still some mistakes for $\alpha=p=1$. To be specific, it shows in equation (2.9) of [23] that $\int_{1}^{u} y^{(1 /(\alpha-2))} \log y d y$ $\leq C u^{(1 /(\alpha-1))} \log u$, where we know it is wrong when $\alpha=1$; furthermore, the formula $\sum_{n=1}^{m}\left(\log n / n^{\alpha}\right) \leq C\left(\log m / n^{\alpha-1}\right)$ in equation (2.11) of [23] is yet invalid for $\alpha=1$. One goal of this work is to further investigate the Baum-Katz type convergence theorem such as complete convergence and complete moment convergence under a much broad dependence assumption and obtain some new results including the interesting case $\alpha=p=1$. Moreover, to the best of my knowledge, there was no paper investigating the limit properties of random vectors with random coefficients in Hilbert spaces. Therefore, the work will mainly focus on this topic to obtain some results which were not established before.

In this paper, some new results on the weak law of large numbers, strong law of large numbers, complete convergence, and complete moment convergence for linear process of $H$-valued $m$-coordinatewise negatively associated ( $m$-CNA, in short) random vectors with random coefficients are established successfully. The results improve and generalise the corresponding ones of Hien and Thanh [9], Huan et al. [18], Huan [20], Ko [21], and Ko [23].

In what follows, let $C$ denote a generic positive constant whose value may vary in different lines. $x_{+}=\max \{x, 0\}$ and $I(A)$ implies the indicator function of the set $A$. $\mathbb{Z}=\{\ldots,-2,-1,0,1,2, \ldots\}$ represents the set of integers.

The paper is organized as follows. Section 2 gives some preliminary definitions and lemmas. Section 3 presents the main results and their proofs. Section 4 contains the conclusion of the paper.

\section{Preliminaries}

In this section, we will present some concepts and important lemmas as below.
Definition 1 (see [18]). A sequence $\left\{X_{i}, i \geq 1\right\}$ of random vectors taking values in $H$ is said to be CNA if, for each $j \in \mathscr{B}$, the sequence $\left\{X_{i}^{(j)}, i \geq 1\right\}$ of random variables is NA, where $X_{i}^{(j)}=\left\langle X_{i}, e_{j}\right\rangle$. Inspired by Hu et al. [3] and Huan et al. [18], we introduce the concept of $m$-CNA random vectors in Hilbert space as follows.

Definition 2. Let $m \geq 1$ be a given integer. A sequence $\left\{X_{n}, n \geq 1\right\}$ of random variables is said to be $m$-CNA if, for any $n \geq 2$ and any $\left(i_{1}, i_{2}, \ldots, i_{n}\right)$ such that $\left|i_{j}-i_{k}\right| \geq m$ for all $1 \leq k \neq j \leq n$, we have that $\left(X_{i_{1}}, X_{i_{2}}, \ldots, X_{i_{n}}\right)$ are CNA. Obviously, if $m=1$, then $m$-CNA random vectors is CNA. Hence, the concept of $m$-CNA random vectors is a natural extension of that of CNA random vectors. We also present an example of $m$-CNA random vectors which are not CNA, as follows.

Example 1. Let $\left\{\xi_{i}, i \geq 1\right\}$ be a sequence of independent random vectors, where $\xi_{i}$ follows the standard multivariate normal distribution for each $i \geq 1$. Take $X_{i}=\xi_{i}+\theta_{1} \xi_{i+1}-$ $\theta_{2} \xi_{i+2}$ for each $i \geq 1$, where $0<\theta_{1}, \theta_{2}<1$. Then, it is easy to check that, for any $j \in \mathscr{B}$ and $i \geq 1, \operatorname{Cov}\left(X_{i}^{(j)}, X_{i+1}^{(j)}\right)=\theta_{1}(1-$ $\left.\theta_{2}\right)>0$ but $\operatorname{Cov}\left(X_{i}^{(j)}, X_{i+2}^{(j)}\right)=-\theta_{2}<0$ and $\operatorname{Cov}\left(X_{i}^{(j)}, X_{i+1}^{(j)}\right)=$ 0 for $l \geq 3$. That is to say, $\left\{X_{i}, i \geq 1\right\}$ is a sequence of $m$-CNA random vectors with $m=2$. We also introduce the following concept of the linear process of random vectors in Hilbert spaces with random coefficients.

Definition 3. Assume that $\left\{X_{i},-\infty<i<\infty\right\}$ is a sequence of $H$-valued random vectors and $\left\{A_{i},-\infty<i<\infty\right\}$ is a sequence of random variables. The sequence $\left\{Y_{t}, t \geq 1\right\}$ of random vectors is said to be linear process with random coefficients if

$$
Y_{t}=\sum_{i=-\infty}^{\infty} A_{i} X_{t-i} .
$$

The following concept is often used in the literature while dealing with the convergence theorems of random vectors in Hilbert spaces.

Definition 4. A sequence $\left\{X_{n}, n \geq 1\right\}$ is said to be coordinatewise weakly upper bounded by $X$ if there exists a positive constant $C$ such that $\sup _{n \geq 1} n^{-1} \sum_{i=1}^{n} P\left(\left|X_{i}^{(j)}\right|>x\right) \leq C P\left(\left|X_{i}^{(j)}\right|>x\right)$, for all $j \in \mathscr{B}$ and $x \geq 0$.

Lemma 1. Let $\left\{X_{i},-\infty<i<\infty\right\}$ be a sequence of $m$-CNA random vectors. If $\left\{f_{i}(\cdot),-\infty<i<\infty\right\}$ is a sequence of coordinatewise nondecreasing (or nonincreasing) continuous real functions, then $\left\{\sum_{j \in \mathscr{B}} f_{i}\left(X_{i}^{(j)}\right) e_{j},-\infty<i<\infty\right\}$ is still a sequence of $m$-CNA random vectors.

Proof. It follows from Definitions 1 and 2 that, for any $n \geq 2$ and any $\left(i_{1}, i_{2}, \ldots, i_{n}\right)$ taking values in $\mathbb{Z}$ such that $\mid i_{j}-$ $i_{k} \mid \geq m$ for all $1 \leq k \neq j \leq n,\left\{X_{i 1}^{(j)}, X_{i 2}^{(j)}, \ldots, X_{i n}^{(j)}\right\}$ is a sequence of NA random variables for each $j \in \mathscr{B}$. By Lemma 2.1 of [24], one can see that $\left\{f_{i 1}\left(X_{i 1}^{(j)}\right), f_{i 2}\left(X_{i 2}^{(j)}\right), \ldots, f_{i n}\left(X_{i n}^{(j)}\right)\right\}$ is still a sequence of 
NA random variables for each $j \in \mathscr{B}$. Hence, by Definitions 1 and 2 again, it follows that $f_{i_{1}}\left(X_{i_{1}}\right), f_{i_{2}}\left(X_{i_{2}}\right), \ldots, f_{i_{n}}\left(X_{i_{n}}\right)$ are CNA, i.e., $\left\{\sum_{j \in \mathscr{B}} f_{i}\left(X_{i}^{(j)}\right),-\infty<i<\infty\right\}$ is $m$-CNA.

Lemma 2 (see [18]). Let $\left\{X_{n}, n \geq 1\right\}$ be a sequence of $H$-valued CNA random vectors with zero mean and $E\left\|X_{n}\right\|^{2}<\infty$, for all $n \geq 1$. Then,

$$
E \max _{1 \leq k \leq n}\left\|\sum_{i=1}^{k} X_{i}\right\|^{2} \leq 2 \sum_{i=1}^{n} E\left\|X_{i}\right\|^{2} .
$$

Lemma 3. Let $\left\{X_{n}, n \geq 1\right\}$ be a sequence of $H$-valued $m$-CNA random vectors with zero mean and $E\left\|X_{n}\right\|^{2}<\infty$ for all $n \geq 1$. Then,

$$
E \max _{1 \leq k \leq n}\left\|\sum_{i=1}^{k} X_{i}\right\|^{2} \leq 2 m \sum_{i=1}^{n} E\left\|X_{i}\right\|^{2}
$$

Proof. Notice that

$$
\max _{1 \leq k \leq n}\left\|\sum_{i=1}^{k} X_{i}\right\| \leq \sum_{l=0}^{m-1} \max _{k \geq 1, m k \leq n}\left\|\sum_{i=1}^{k} X_{m i+l}\right\|,
$$

where we can define without loss of generality that $X_{0}=0$. From Definition 1, we see that $\left\{X_{m i+l}, i \geq 0\right\}$ is CNA for each $(l=1,2, \ldots, m)$. Hence, by $c_{r}$ inequality and Lemma 2 , we have

$$
\begin{aligned}
E \max _{1 \leq k \leq n}\left\|\sum_{i=1}^{k} X_{i}\right\|^{2} & \leq m \sum_{l=0}^{m-1} E \max _{k \geq 1, m k \leq n}\left\|\sum_{i=1}^{k} X_{m i+l}\right\|^{2} \\
& \leq 2 m \sum_{l=0}^{m-1} \sum_{i \geq 0, m i \leq n} E\left\|X_{m i+l}\right\|^{2} \\
& \leq 2 m \sum_{i=0}^{n} E\left\|X_{i}\right\|^{2} .
\end{aligned}
$$

Lemma 4. Let $\left\{X_{i},-\infty<i<\infty\right\}$ be a sequence of $H$-valued $m$-CNA random vectors with zero mean and $E\left\|X_{i}\right\|^{2}<\infty$ for all $-\infty<i<\infty$. If $\left\{A_{i},-\infty<i<\infty\right\}$ is a sequence of random variables independent of $\left\{X_{i},-\infty<i<\infty\right\}$ and $E\left(\sum_{i=-\infty}^{\infty} A_{i}\right)^{2}<\infty$, then

$$
E \max _{1 \leq k \leq n}\left\|\sum_{l=-\infty}^{\infty} \sum_{i=1-l}^{k-l} A_{i} X_{l}\right\|^{2} \leq C \sup _{-\infty<i<\infty} \sum_{l=1-i}^{n-i} E\left\|X_{l}\right\|^{2} .
$$

Proof. It follows by Hölder inequality and Lemma 3 that

$$
\begin{aligned}
E \max _{1 \leq k \leq n}\left\|\sum_{l=-\infty}^{\infty} \sum_{i=1-l}^{k-l} A_{i} X_{l}\right\|^{2} & =E \max _{1 \leq k \leq n}\left\|\sum_{i=-\infty}^{\infty} A_{i} \sum_{l=1-i}^{k-i} X_{l}\right\|^{2} \\
& \leq E\left[\sum_{i=-\infty}^{\infty}\left|A_{i}\right| \max _{1 \leq k \leq n}\left\|\sum_{l=1-i}^{k-i} X_{l}\right\|\right]^{2} \\
& =E\left[\sum_{i=-\infty}^{\infty}\left|A_{i}\right|^{(1 / 2)} \cdot\left|A_{i}\right|^{(1 / 2)} \max _{1 \leq k \leq n}\left\|\sum_{l=1-i}^{k-i} X_{l}\right\|\right]^{2} \\
& \leq E\left[\left(\sum_{i=-\infty}^{\infty}\left|A_{i}\right|\right)^{(1 / 2)}\left(\sum_{i=-\infty}^{\infty}\left|A_{i}\right| \max _{1 \leq k \leq n}\left\|\sum_{l=1-i}^{k-i} X_{l}\right\|^{2}\right)^{(1 / 2)}\right]^{2} \\
& =\sum_{s=1}^{\infty} E\left[\left(\sum_{i=-\infty}^{\infty}\left|A_{i}\right|\right)\left(\sum_{i=-\infty}^{\infty}\left|A_{i}\right| \max _{1 \leq k \leq n}\left\|\sum_{l=1-i}^{k-i} X_{l}\right\|^{2}\right) I\left(s-1 \leq \sum_{i=-\infty}^{\infty}\left|A_{i}\right|<s\right)\right] \\
& \leq \sum_{s=1}^{\infty} s E\left[\sum_{i=-\infty}^{\infty}\left|A_{i}\right| I\left(s-1 \leq \sum_{i=-\infty}^{\infty}\left|A_{i}\right|<s\right)\right] E \max _{1 \leq k \leq n}\left\|\sum_{l=1-i}^{k-i} X_{l}\right\|^{2} \\
& \leq 2 m \sup _{-\infty<i<\infty} \sum_{l=1-i}^{n-i} E\left\|X_{l}\right\|^{2}\left[1+2 \sum_{s=2}^{\infty} E\left(\sum_{i=-\infty}^{\infty}\left|A_{i}\right|\right)^{2} I\left(s-1 \leq \sum_{i=-\infty}^{\infty}\left|A_{i}\right|<s\right)\right] \\
& \leq C \sup _{-\infty<i<\infty}^{n-i} \sum_{l=1-i} E\left\|X_{l}\right\|^{2} .
\end{aligned}
$$

The proof is therefore complete. 
Lemma 5 (see [25]). Let $\left\{X_{n}, n \geq 1\right\}$ be a sequence of random variables satisfying $n^{-1} \sum_{i=1}^{n} P\left(\left|Z_{i}\right|>x\right) \leq C P(|Z|>x)$ for a random variable $Z$ and any $x \geq 0$. Then, for any $a>0$ and $b>0$, there exist some positive constants $c_{1}$ and $c_{2}$ such that

$$
\begin{aligned}
& n^{-1} \sum_{i=1}^{n} E\left|Z_{i}\right|^{a} I\left(\left|Z_{i}\right|>b\right) \leq c_{1} E|Z|^{a} I(|Z|>b), \\
& n^{-1} \sum_{i=1}^{n} E\left|X_{i}\right|^{a} I\left(\left|Z_{i}\right| \leq b\right) \leq c_{2}\left[E|Z|^{a} I(|Z| \leq b)+b^{a} P(|Z|>b)\right] .
\end{aligned}
$$

Following the method of Lemma 2.3 in [26], we can obtain the following inequality in Hilbert spaces.
Lemma 6. Let $\left\{Y_{i}, 1 \leq i \leq n\right\}$ and $\left\{Z_{i}, 1 \leq i \leq n\right\}$ be two sequences of random vectors. Then, for any $q>1, \varepsilon>0$, and $a>0$, the following inequality holds:

$$
E\left(\max _{1 \leq k \leq n}\left\|\sum_{i=1}^{k}\left(Y_{i}+Z_{i}\right)\right\|-\varepsilon a\right)^{+} \leq\left(\frac{1}{\varepsilon^{q}}+\frac{1}{q-1}\right) a^{1-q} E\left(\max _{1 \leq k \leq n}\left\|\sum_{i=1}^{k} Y_{i}\right\|^{q}\right)+E \max _{1 \leq k \leq n}\left\|\sum_{i=1}^{k} Z_{i}\right\|
$$

\section{Main Results and Their Proofs}

In this section, we will present our main results. The first one is the weak law of large numbers for linear process $\left\{Y_{t}, t \geq 1\right\}$ of $m$-CNA random vectors in Hilbert spaces with random coefficients.

Theorem 1. Let $\left\{X_{i},-\infty<i<\infty\right\}$ be a sequence of zero mean $H$-valued $m$-CNA random vectors coordinatewise weakly upper bounded by a random vector $X$. Suppose that $\left\{A_{i},-\infty<i<\infty\right\}$ is a sequence of random variables independent of $\left\{X_{i},-\infty<i<\infty\right\}$ and $E\left(\sum_{i=-\infty}^{\infty} A_{i}\right)^{2}<\infty$. Assume further $\sum_{j \in \mathscr{B}} E\left|X^{(j)}\right| I\left(\left|X^{(j)}\right|>n\right) \longrightarrow 0$ as $n \longrightarrow \infty$ if the cardinality $|\mathscr{B}|<\infty$ or $\sum_{j \in \mathscr{B}} E\left|X^{(j)}\right|<\infty$ if $|\mathscr{B}|=\infty$. Then,

$$
\frac{1}{n} \sum_{t=1}^{n} Y_{t} \stackrel{P}{\longrightarrow} 0, \quad \text { as } n \longrightarrow \infty
$$

Proof. For each $-\infty<i<\infty$ and $j \in \mathscr{B}$, denote

$$
\begin{aligned}
X_{n i}^{(j)} & =-n I\left(X_{i}^{(j)}<-n\right)+X_{i}^{(j)} I\left(\left|X_{i}^{(j)}\right| \leq n\right)+n I\left(X_{i}^{(j)}>n\right) \\
Z_{n i}^{(j)} & =X_{i}^{(j)}-X_{n i}^{(j)}=\left(X_{i}^{(j)}+n\right) I\left(X_{i}^{(j)}<-n\right)+\left(X_{i}^{(j)}-n\right) I\left(X_{i}^{(j)}>n\right) \\
X_{n i} & =\sum_{j \in \mathscr{B}} X_{n i}^{(j)} e_{j} \\
Z_{n i} & =\sum_{j \in \mathscr{B}} Z_{n i}^{(j)} e_{j} .
\end{aligned}
$$

Note that

$$
\begin{aligned}
\sum_{t=1}^{n} Y_{t} & =\sum_{t=1}^{n} \sum_{i=-\infty}^{\infty} A_{i} X_{t-i}=\sum_{l=-\infty}^{\infty} \sum_{i=1-l}^{n-l} A_{i} X_{l} \\
& =\sum_{l=-\infty}^{\infty} \sum_{i=1-l}^{n-l} A_{i}\left(X_{n l}-E X_{n l}\right)+\sum_{l=-\infty}^{\infty} \sum_{i=1-l}^{n-l} A_{i}\left(Z_{n l}-E Z_{n l}\right) \\
& =\sum_{l=-\infty}^{\infty} \sum_{i=1-l}^{n-l} A_{i}\left(X_{n l}-E X_{n l}\right)+\sum_{t=1}^{n} \sum_{i=-\infty}^{\infty} A_{i}\left(Z_{n, t-i}-E Z_{n, t-i}\right) .
\end{aligned}
$$


It is sufficient to prove that, for any $\varepsilon>0$,

$$
\begin{gathered}
P\left(\left\|\sum_{l=-\infty}^{\infty} \sum_{i=1-l}^{n-l} A_{i}\left(X_{n l}-E X_{n l}\right)\right\|>n \varepsilon\right) \longrightarrow 0, \\
P\left(\left\|\sum_{t=1}^{n} \sum_{i=-\infty}^{\infty} A_{i}\left(Z_{n, t-i}-E Z_{n, t-i}\right)\right\|>n \varepsilon\right) \longrightarrow 0 .
\end{gathered}
$$

It follows from Lemma 1 that $\left\{X_{n i},-\infty<i \leq \infty\right\}$ is still a sequence of $m$-CNA random vectors. Furthermore, it is easy to check that, as $n \longrightarrow \infty$,

$$
\begin{aligned}
& n^{-1} \sum_{j \in \mathscr{B}} \int_{0}^{1} x P\left(\left|X^{(j)}\right|>x\right) \mathrm{d} x \leq n^{-1} \sum_{j \in \mathscr{B}} E\left|X^{(j)}\right| \longrightarrow 0, \quad \text { if }|\mathscr{B}|=\infty, \\
& n^{-1} \sum_{j \in \mathscr{B}} \int_{0}^{1} x P\left(\left|X^{(j)}\right|>x\right) \mathrm{d} x \leq n^{-1}|\mathscr{B}| \longrightarrow 0, \quad \text { if }|\mathscr{B}|<\infty .
\end{aligned}
$$

Hence, we obtain by Chebyshev inequality, $E\left(\sum_{i=-\infty}^{\infty} A_{i}\right)^{2}<\infty$, Lemmas 4 and 5 , and integration by parts that

$$
\begin{aligned}
P\left(\left\|\sum_{l=-\infty}^{\infty} \sum_{i=1-l}^{n-l} A_{i}\left(X_{n l}-E X_{n l}\right)\right\|>n \varepsilon\right) & C n^{-2} E\left\|\sum_{l=-\infty}^{\infty} \sum_{i=1-l}^{n-l} A_{i}\left(X_{n l}-E X_{n l}\right)\right\|^{2} \\
& \leq C n^{-2} \sup _{-\infty<i<\infty} \sum_{l=1-i}^{n-i} E\left\|\left(X_{n l}-E X_{n l}\right)\right\|^{2} \\
& \leq C n^{-2} \sup _{-\infty<i<\infty} \sum_{l=1-i}^{n-i}\left\{\sum_{j \in \mathscr{B}}\left[E\left|X_{l}^{(j)}\right|^{2} I\left(\left|X_{l}^{(j)}\right| \leq n\right)+n^{2} P\left(\left|X_{l}^{(j)}\right|>n\right)\right]\right\} \\
& \leq C n^{-1} \sum_{j \in \mathscr{B}}\left[E\left|X^{(j)}\right|^{2} I\left(\left|X^{(j)}\right| \leq n\right)+n^{2} P\left(\left|X^{(j)}\right|>n\right)\right] \\
& =C n^{-1} \sum_{j \in \mathscr{B}} \int_{0}^{n} x\left(P\left|X^{(j)}\right|>x\right) d x \\
& =C n^{-1} \sum_{j \in \mathscr{B}}^{n-1} \sum_{k=0}^{k} \int_{k}^{k+1} x\left(P\left|X^{(j)}\right|>x\right) d x \\
& \leq C n^{-1} \sum_{j \in \mathscr{B}} \int_{0}^{1} x P\left(\left|X^{(j)}\right|>x\right) d x+C n^{-1} \sum_{j \in \mathscr{B}} \sum_{k=1}^{n}\left[(k+1)^{2}-k^{2}\right] P\left(\left|X^{(j)}\right|>k\right) \\
& \leq C n^{-1} \sum_{j \in \mathscr{B}} \int_{0}^{1} x P\left(\left|X^{(j)}\right|>x\right) d x+C n^{-1} \sum_{k=1}^{n}\left(\sum_{j \in \mathscr{B}} k P\left(\left|X^{(j)}\right|>k\right)\right) \\
& \leq C n^{-1} \sum_{j \in \mathscr{B}} \int_{0}^{1} x P\left(\left|X^{(j)}\right|>x\right) d x+C n^{-1} \sum_{k=1}^{n}\left(\sum_{j \in \mathscr{B}} E\left|X^{(j)}\right| I\left(\left|X^{(j)}\right|>k\right)\right)
\end{aligned}
$$

which converges to 0 as $n \longrightarrow \infty$, and thus, equation (14) holds true. On the contrary, we have, by Markov inequality, Lemma 5, and Jensen inequality, that 


$$
\begin{aligned}
P\left(\left\|\sum_{t=1}^{n} \sum_{i=-\infty}^{\infty} A_{i}\left(Z_{n, t-i}-E Z_{n, t-i}\right)\right\|>n \varepsilon\right) & \leq C n^{-1} E\left\|\sum_{t=1}^{n} \sum_{i=-\infty}^{\infty} A_{i}\left(Z_{n, t-i}-E Z_{n, t-i}\right)\right\| \\
& \leq C n^{-1} \sum_{t=1}^{n} \sum_{i=-\infty}^{\infty} E\left|A_{i}\right| E\left\|Z_{n, t-i}-E Z_{n, t-i}\right\| \\
& \leq C E\left(\sum_{i=-\infty}^{\infty}\left|A_{i}\right|\right) \sum_{j \in \mathscr{B}} n^{-1} \sum_{t=1}^{n} E\left|X_{t-i}^{(j)}\right| I\left(\left|X_{t-i}^{(j)}\right|>n\right) \\
& \leq C\left[E\left(\sum_{i=-\infty}^{\infty}\left|A_{i}\right|\right)^{2}\right]_{j \in \mathscr{B}}^{(1 / 2)} E\left|X^{(j)}\right| I\left(\left|X^{(j)}\right|>n\right),
\end{aligned}
$$

which obtains equation (15) as $n \longrightarrow \infty$ by the assumption of Theorem 1, and the proof is thus complete.

Remark 1. Hien and Thanh [9] obtained the weak law of large numbers for NA random vectors under the moment condition $\sum_{j \in \mathscr{B}} E\left|X^{(j)}\right|<\infty$. Contrasting to Corollary 2.5 of Hien and Thanh [9], Theorem 1 not only extends the assumption of NA random vectors to linear process of $m$-CNA random vectors with random coefficients but also improves the moment condition when $|\mathscr{B}|<\infty$.

Theorem 2. Let $1<p<2$ and $\alpha p \geq 1$. Let $\left\{X_{i},-\infty<i<\infty\right\}$ be a sequence of zero mean $H$-valued $m$-CNA random vectors coordinatewise weakly upper bounded by a random vector $X$ with $\sum_{j \in \mathscr{B}} E\left|X^{(j)}\right|^{p}<\infty$. Suppose that $\left\{A_{i},-\infty<i<\infty\right\}$ is a sequence of random variables independent of $\left\{X_{i},-\infty<i<\infty\right\}$ and $E\left(\sum_{i=-\infty}^{\infty} A_{i}\right)^{2}<\infty$. Then, for any $\varepsilon>0$,

$$
\sum_{n=1}^{\infty} n^{\alpha p-\alpha-2} E\left(\max _{1 \leq k \leq n}\left\|\sum_{t=1}^{k} Y_{t}\right\|-\varepsilon n^{\alpha}\right)_{+}<\infty
$$

and thus,

$$
\sum_{n=1}^{\infty} n^{\alpha p-2} P\left(\max _{1 \leq k \leq n}\left\|\sum_{t=1}^{k} Y_{t}\right\|>\varepsilon n^{\alpha}\right)<\infty
$$

Proof. Define for each $-\infty<i<\infty$ and $j \in \mathscr{B}$ that

$$
\begin{aligned}
W_{n i}^{(j)} & =-n I\left(X_{i}^{(j)}<-n^{\alpha}\right)+X_{i}^{(j)} I\left(\left|X_{i}^{(j)}\right| \leq n^{\alpha}\right)+n I\left(X_{i}^{(j)}>n^{\alpha}\right), \\
T_{n i}^{(j)} & =X_{i}^{(j)}-W_{n i}^{(j)}=\left(X_{i}^{(j)}+n^{\alpha}\right) I\left(X_{i}^{(j)}<-n^{\alpha}\right)+\left(X_{i}^{(j)}-n^{\alpha}\right) I\left(X_{i}^{(j)}>n^{\alpha}\right), \\
W_{n i} & =\sum_{j \in \mathscr{B}} W_{n i}^{(j)} e_{j}, \\
T_{n i} & =\sum_{j \in \mathscr{B}} T_{n i}^{(j)} e_{j} .
\end{aligned}
$$

Similar to the argument of equation (13), we have that, for each $1 \leq k \leq n$,

$$
\sum_{t=1}^{k} Y_{t}=\sum_{l=-\infty}^{\infty} \sum_{i=1-l}^{k-l} A_{i} X_{l}=\sum_{l=-\infty}^{\infty} \sum_{i=1-l}^{k-l} A_{i}\left(W_{n l}-E W_{n l}\right)+\sum_{t=1}^{k} \sum_{i=-\infty}^{\infty} A_{i}\left(T_{n, t-i}-E T_{n, t-i}\right)
$$

On the one hand, noting that $\left\{W_{n i},-\infty<i \leq \infty\right\}$ is still a sequence of $m$-CNA random vectors by Lemma 1 , we obtain by Lemmas 4 and 5 that 


$$
\begin{aligned}
& \sum_{n=1}^{\infty} n^{\alpha p-2 \alpha-2} E \max _{1 \leq k \leq n}|| \sum_{l=-\infty}^{\infty} \sum_{i=1-l}^{k-l} A_{i}\left(W_{n l}-E W_{n l}\right) \|^{2} \\
& \leq C \sum_{n=1}^{\infty} n^{\alpha p-2 \alpha-2} \sup _{-\infty<i<\infty} \sum_{l=1-i}^{n-i}\left\{\sum_{j \in \mathscr{B}}\left[E\left|X_{l}^{(j)}\right|^{2} I\left(\left|X_{l}^{(j)}\right| \leq n^{\alpha}\right)+n^{2 \alpha} P\left(\left|X_{l}^{(j)}\right|>n^{\alpha}\right)\right]\right\} \\
& \leq C \sum_{j \in \mathscr{B}} \sum_{n=1}^{\infty} n^{\alpha p-2 \alpha-1} E\left|X^{(j)}\right|^{2} I\left(\left|X^{(j)}\right| \leq n^{\alpha}\right)+C \sum_{j \in \mathscr{B}} \sum_{n=1}^{\infty} n^{\alpha p-1} P\left(\left|X^{(j)}\right|>n^{\alpha}\right) \\
& \leq C \sum_{j \in \mathscr{B}} \sum_{n=1}^{\infty} n^{\alpha p-2 \alpha-1} \sum_{l=1}^{n} E\left|X^{(j)}\right|^{2} I\left((l-1)^{\alpha}<\left|X^{(j)}\right| \leq l^{\alpha}\right)+C \sum_{j \in \mathscr{B}} E\left|X^{(j)}\right|^{p} \\
& \leq C \sum_{j \in \mathscr{B}} \sum_{l=1}^{\infty} l^{\alpha p-2 \alpha} E\left|X^{(j)}\right|^{2} I\left((l-1)^{\alpha}<\left|X^{(j)}\right| \leq l^{\alpha}\right)+C \sum_{j \in \mathscr{B}} E\left|X^{(j)}\right|^{p} \\
& \leq C \sum_{j \in \mathscr{B}} E\left|X^{(j)}\right|^{p}<\infty .
\end{aligned}
$$

On the other hand, it follows from Lemma 5 and Jensen inequality that

$$
\begin{aligned}
& \sum_{n=1}^{\infty} n^{\alpha p-\alpha-2} E \max _{1 \leq k \leq n}\left\|\sum_{t=1}^{k} \sum_{i=-\infty}^{\infty} A_{i}\left(T_{n, t-i}-E T_{n, t-i}\right)\right\| \\
& \leq C \sum_{n=1}^{\infty} n^{\alpha p-\alpha-2} \sum_{t=1}^{n} \sum_{i=-\infty}^{\infty} E\left|A_{i}\right| E\left\|T_{n, t-i}-E T_{n, t-i}\right\| \\
& \leq C \sum_{n=1}^{\infty} n^{\alpha p-\alpha-1} E\left(\sum_{i=-\infty}^{\infty}\left|A_{i}\right|\right) \sum_{j \in \mathscr{B}} n^{-1} \sum_{t=1}^{n} E\left|X_{t-i}^{(j)}\right| I\left(\left|X_{t-i}^{(j)}\right|>n^{\alpha}\right) \\
& \leq C \sum_{j \in \mathscr{B}} \sum_{n=1}^{\infty} n^{\alpha p-\alpha-1}\left[E\left(\sum_{i=-\infty}^{\infty}\left|A_{i}\right|\right)^{2}\right]^{(1 / 2)} E\left|X^{(j)}\right| I\left(\left|X^{(j)}\right|>n^{\alpha}\right) \\
& \leq C \sum_{j \in \mathscr{B}} \sum_{l=1}^{\infty} E\left|X^{(j)}\right| I\left(l^{\alpha}<\left|X^{(j)}\right| \leq(l+1)^{\alpha}\right) \sum_{n=1}^{l} n^{\alpha p-\alpha-1} \\
& \leq C \sum_{j \in \mathscr{B}} \sum_{l=1}^{\infty} l^{\alpha p-\alpha} E\left|X^{(j)}\right| I\left(l^{\alpha}<\left|X^{(j)}\right| \leq(l+1)^{\alpha}\right) \\
& \leq C \sum_{j \in \mathscr{B}} E\left|X^{(j)}\right|^{p}<\infty .
\end{aligned}
$$

Hence, it follows from Lemma 6 (with $q=2$ ) and equations (22)-(24) that 


$$
\begin{aligned}
& \sum_{n=1}^{\infty} n^{\alpha p-\alpha-2} E\left(\max _{1 \leq k \leq n}\left\|\sum_{t=1}^{k} Y_{t}\right\|-\varepsilon n^{\alpha}\right)_{+} \\
& \leq C \sum_{n=1}^{\infty} n^{\alpha p-2 \alpha-2} E \max _{1 \leq k \leq n}\left\|\sum_{l=-\infty}^{\infty} \sum_{i=1-l}^{k-l} A_{i}\left(W_{n l}-E W_{n l}\right)\right\|^{2}+\sum_{n=1}^{\infty} n^{\alpha p-\alpha-2} E \max _{1 \leq k \leq n}\left\|\sum_{t=1}^{k} \sum_{i=-\infty}^{\infty} A_{i}\left(T_{n, t-i}-E T_{n, t-i}\right)\right\| \\
& \leq C \sum_{j \in \mathscr{B}} E\left\|X^{(j)}\right\|^{p}<\infty
\end{aligned}
$$

which obtains equation (19). Now, we prove equation (20). It follows from equation (19) that

$$
\begin{aligned}
& \infty>\sum_{n=1}^{\infty} n^{\alpha p-\alpha-2} E\left(\max _{1 \leq k \leq n}\left\|\sum_{t=1}^{k} Y_{t}\right\|-\varepsilon n^{\alpha}\right)_{+} \\
& \geq \sum_{n=1}^{\infty} n^{\alpha p-\alpha-2} \int_{0}^{\varepsilon^{\alpha}} P\left(\max _{1 \leq k \leq n}\left\|\sum_{t=1}^{k} Y_{t}\right\|-\varepsilon n^{\alpha}>s\right) \mathrm{d} s \\
& \geq \varepsilon \sum_{n=1}^{\infty} n^{\alpha p-2} P\left(\max _{1 \leq k \leq n}\left\|\sum_{t=1}^{k} Y_{t}\right\|>2 \varepsilon n^{\alpha}\right),
\end{aligned}
$$

which combining with the arbitrariness of $\varepsilon$ gets equation (20). The proof is complete.

Remark 2. Huan et al. [18] and Huan [20] established equation (20) for CNA random vectors, respectively, for $1 \leq p<2, \alpha p>1$, and $1<p<2, \alpha p=1$. Although Ko [21] extended the result of Huan et al. [18] to complete moment convergence, it only holds for $1<p<2, \alpha p>1$, as illustrated in Section 1. Hence, Theorem 2 improves and extends the results of Huan et al. [18], Huan [20], and Ko [21] from CNA random vectors to linear process of $m$-CNA random vectors with random coefficients. By Theorem 2, we can easily get the following conclusion.

Corollary 1. Under the conditions of Theorem 2, if $\alpha p>1$, we have that, for any $\varepsilon>0$,

$$
\sum_{n=1}^{\infty} n^{\alpha p-2} E\left(\sup _{k \geq n} k^{-\alpha}\left\|\sum_{t=1}^{k} Y_{t}\right\|-\varepsilon\right)_{+}<\infty,
$$

and thus,

$$
\sum_{n=1}^{\infty} n^{\alpha p-2} P\left(\sup _{k \geq n} k^{-\alpha}\left\|\sum_{t=1}^{k} Y_{t}\right\|>\varepsilon\right)<\infty .
$$

Proof. It follows from Theorem 2 that

$$
\begin{aligned}
\sum_{n=1}^{\infty} n^{\alpha p-2} E\left(\sup _{k \geq n} k^{-\alpha}\left\|\sum_{t=1}^{k} Y_{t}\right\|-\varepsilon\right)_{+} & =\sum_{l=1}^{\infty} \sum_{2^{l-1} \leq n<2^{l}} n^{\alpha p-2} E\left(\sup _{k \geq n} k^{-\alpha}\left\|\sum_{t=1}^{k} Y_{t}\right\|_{+}\right)_{+} \\
& \leq C \sum_{l=1}^{\infty} 2^{l(\alpha p-1)} E\left(\sup _{k \geq 2^{l-1}} k^{-\alpha}\left\|\sum_{t=1}^{k} Y_{t}\right\|-\varepsilon\right)_{+} \\
& \leq C \sum_{s=1}^{\infty} E\left(\max _{2^{s-1} \leq k<2^{s}} k^{-\alpha}\left\|\sum_{t=1}^{k} Y_{t}\right\|-\varepsilon \sum_{+=1}^{s} 2^{l(\alpha p-1)}\right. \\
& \leq C \sum_{s=1}^{\infty} 2^{s(\alpha p-1)} E\left(\max _{2^{s-1} \leq k<2^{s}} 2^{-\alpha(s-1)}\left\|\sum_{t=1}^{k} Y_{t}\right\|_{-\varepsilon}\right)_{+} \\
& \leq C \sum_{s=1}^{\infty} 2^{s(\alpha p-\alpha-1)} E\left(\max _{1 \leq k<2^{s}}\left\|\sum_{t=1}^{k} Y_{t}\right\|-\varepsilon 2^{-\alpha} 2^{\alpha s}\right)_{+} \\
& \leq C \sum_{s=0}^{\infty} \sum_{2^{s} \leq n<2^{s+1}} n^{\alpha p-\alpha-2} E\left(\max _{1 \leq k<2^{s}}\left\|\sum_{t=1}^{k} Y_{t}\right\|-\varepsilon 2^{-\alpha} 2^{\alpha s}\right)_{+} \\
& \leq C \sum_{n=1}^{\infty} n^{\alpha p-\alpha-2} E\left(\max _{1 \leq k \leq n}\left\|\sum_{t=1}^{k} Y_{t}\right\|-\varepsilon 2^{-2 \alpha} n^{\alpha}\right)_{+}^{<\infty}
\end{aligned}
$$

Furthermore, similar to the proof of equation (20), we have that 


$$
\begin{aligned}
& \infty>\sum_{n=1}^{\infty} n^{\alpha p-2} E\left(\sup _{k \geq n} k^{-\alpha}\left\|\sum_{t=1}^{k} Y_{t}\right\|-\varepsilon\right)_{+} \\
& \geq \sum_{n=1}^{\infty} \sum_{n=1}^{\infty} n^{\alpha p-2} \int_{0}^{\varepsilon} P\left(\sup _{k \geq n} k^{-\alpha}\left\|\sum_{t=1}^{k} Y_{t}\right\|-\varepsilon>s\right) \mathrm{d} s \\
& \geq \varepsilon \sum_{n=1}^{\infty} n^{\alpha p-2} P\left(\sup _{k \geq n} k^{-\alpha}\left\|\sum_{t=1}^{k} Y_{t}\right\|>2 \varepsilon\right) .
\end{aligned}
$$

The proof is complete.

For $p=1$, we can obtain the following result.

Theorem 3. Let $\alpha \geq 1$. Let $\left\{X_{i},-\infty<i<\infty\right\}$ be a sequence of zero mean $H$-valued $m$-CNA random vectors coordinatewise weakly upper bounded by a random vector $X$ with $\sum_{j \in \mathscr{B}} E\left|X^{(j)}\right| \ln ^{\delta+1}\left(1+\left|X^{(j)}\right|\right)<\infty$ for some $\delta \geq 0$. Suppose that $\left\{A_{i},-\infty<i<\infty\right\}$ is a sequence of random variables independent of $\left\{X_{i},-\infty<i<\infty\right\}$ and $E\left(\sum_{i=-\infty}^{\infty} A_{i}\right)^{2}<\infty$. Then, for any $\varepsilon>0$,

$$
\sum_{n=1}^{\infty} n^{-2} \ln ^{\delta} n E\left(\max _{1 \leq k \leq n}\left\|\sum_{t=1}^{k} Y_{t}\right\|-\varepsilon n^{\alpha}\right)_{+}<\infty,
$$

and thus,

$$
\sum_{n=1}^{\infty} n^{\alpha-2} \ln ^{\delta} n P\left(\max _{1 \leq k \leq n}\left\|\sum_{t=1}^{k} Y_{t}\right\|>\varepsilon n^{\alpha}\right)<\infty .
$$

Proof. We still use the notations and method in the proof of Theorem 2. Similar to the argument of equation (23), we have

$$
\begin{aligned}
& \sum_{n=1}^{\infty} n^{-\alpha-2} \ln ^{\delta} n E \max _{1 \leq k \leq n}|| \sum_{l=-\infty}^{\infty} \sum_{i=1-l}^{k-l} A_{i}\left(W_{n l}-E W_{n l}\right) \|^{2} \\
& \leq C \sum_{j \in \mathscr{B}} \sum_{n=1}^{\infty} n^{-\alpha-1} \ln ^{\delta} n E\left|X^{(j)}\right|^{2} I\left(\left|X^{(j)}\right| \leq n^{\alpha}\right)+C \sum_{j \in \mathscr{B}} \sum_{n=1}^{\infty} n^{\alpha-1} \ln ^{\delta} n P\left(\left|X^{(j)}\right|>n^{\alpha}\right) \\
& \leq C \sum_{j \in \mathscr{B}}^{\infty} \sum_{l=1}^{\infty} l^{-\alpha} \ln ^{\delta} l E\left|X^{(j)}\right|^{2} I\left((l-1)^{\alpha}<\left|X^{(j)}\right| \leq l^{\alpha}\right)+C \sum_{j \in \mathscr{B}}^{\infty} \sum_{l=1}^{\infty} l^{\alpha} \ln ^{\delta} l P\left(l^{\alpha}<\left|X^{(j)}\right| \leq(l+1)^{\alpha}\right) \\
& \leq C \sum_{j \in \mathscr{B}} \sum_{l=1}^{\infty} E\left|X^{(j)}\right|^{2} \cdot\left|X^{(j)}\right|^{-1}\left(\ln \left(1+\left|X^{(j)}\right|\right)^{(1 / \alpha)}\right)^{\delta} I\left((l-1)^{\alpha}<\left|X^{(j)}\right| \leq l^{\alpha}\right) \\
& +C \sum_{j \in \mathscr{B}} \sum_{l=1}^{\infty} E\left|X^{(j)}\right|\left(\ln \left(1+\left|X^{(j)}\right|\right)^{(1 / \alpha)}\right)^{\delta} I\left((l-1)^{\alpha}<\left|X^{(j)}\right| \leq l^{\alpha}\right) \\
& \leq C \sum_{j \in \mathscr{B}} \sum_{l=1}^{\infty} E\left|X^{(j)}\right| \ln ^{\delta}\left(1+\left|X^{(j)}\right|\right) I\left((l-1)^{\alpha}<\left|X^{(j)}\right| \leq l^{\alpha}\right) \\
& \leq C \sum_{j \in \mathscr{B}} E\left|X^{(j)}\right| \ln ^{\delta}\left(1+\left|X^{(j)}\right|\right)<\infty .
\end{aligned}
$$

Furthermore, similar to the argument of equation (24), we have

$$
\begin{aligned}
& \sum_{n=1}^{\infty} n^{-2} \ln ^{\delta} n E \max _{1 \leq k \leq n}|| \sum_{t=1}^{k} \sum_{i=-\infty}^{\infty} A_{i}\left(T_{n, t-i}-E T_{n, t-i}\right) \mid \\
& \leq C \sum_{j \in \mathscr{S}} \sum_{l=1}^{\infty} E\left|X^{(j)}\right| I\left(l^{\alpha}<\left|X^{(j)}\right| \leq(l+1)^{\alpha}\right) \sum_{n=1}^{l} n^{-1} \ln ^{\delta} n \\
& \leq C \sum_{j \in \mathscr{B}} \sum_{l=1}^{\infty} \ln ^{\delta+1} l E\left|X^{(j)}\right| I\left(l^{\alpha}<\left|X^{(j)}\right| \leq(l+1)^{\alpha}\right) \\
& \leq C \sum_{j \in \mathscr{B}} \sum_{l=1}^{\infty} E\left|X^{(j)}\right| \ln ^{\delta+1}\left(1+\left|X^{(j)}\right|\right) I\left(l^{\alpha}<\left|X^{(j)}\right| \leq(l+1)^{\alpha}\right) \\
& \leq C \sum_{j \in \mathscr{S}} E\left|X^{(j)}\right| \ln ^{\delta+1}\left(1+\left|X^{(j)}\right|\right) I\left(\left|X^{(j)}\right|>1\right) \\
& \leq C \sum_{j \in \mathscr{S}} E\left|X^{(j)}\right| \ln ^{\delta+1}\left(1+\left|X^{(j)}\right|\right)<\infty .
\end{aligned}
$$

Hence, by Lemma 1 and equations (22), (33), and (34), we can obtain equation (31). Following the proof of equation (20), we can also get equation (32) by equation (31). The proof is complete.

Remark 3. Ko [23] proved the complete moment convergence for coordinatewise asymptotically almost negatively associated (CAANA) random vectors with $\alpha p=1$ and $(1 / 2)<\alpha \leq 1$. However, as stated in Section 1 , the meaningful case $\alpha=1$ is wrongly proved. Note that Theorem 3 also works if $\alpha=p=1$. Thus, Theorem 3 fills the vacancy and extends it to some more general settings. By Theorems 2 and 3 , one can obtain the following strong law of large numbers for linear process of $m$-CNA random vectors with random coefficients.

Corollary 2. Let $1 \leq p<2$. Let $\left\{X_{i},-\infty<i<\infty\right\}$ be a sequence of zero mean $H$-valued $m$-CNA random vectors 
coordinatewise weakly upper bounded by a random vector $X$ with $\quad \sum_{j \in \mathscr{B}} E\left|X^{(j)}\right|^{p}<\infty \quad$ if $\quad p>1$ or $\sum_{j \in \mathscr{B}} E\left|X^{(j)}\right| \ln \left(1+\left|X^{(j)}\right|\right)<\infty$ if $p=1$. Suppose that $\left\{A_{i},-\infty<i<\infty\right\}$ is a sequence of random variables independent of $\left\{X_{i},-\infty<i<\infty\right\}$ and $E\left(\sum_{i=-\infty}^{\infty} A_{i}\right)^{2}<\infty$. Then, with probability 1 ,

$$
n^{(-1 / p)}\left\|\sum_{t=1}^{k} Y_{t}\right\| \longrightarrow 0, \quad \text { as } n \longrightarrow \infty .
$$

Proof. Let $\alpha=(1 / p)$. It follows from equations (20) and (32) (with $\delta=0$ ) that

$$
\begin{aligned}
& \infty>\sum_{n=1}^{\infty} n^{-1} P\left(\max _{1 \leq k \leq n}\left\|\sum_{t=1}^{k} Y_{t}\right\|>\varepsilon n^{(1 / p)}\right) \\
& =\sum_{l=0}^{\infty} \sum_{2^{l} \leq n<2^{l+1}} n^{-1} P\left(\max _{1 \leq k \leq n}\left\|\sum_{t=1}^{k} Y_{t}\right\|>\varepsilon n^{(1 / p)}\right) \\
& \geq \frac{1}{2} \sum_{l=1}^{\infty} P\left(\max _{1 \leq k \leq 2^{l}}\left\|\sum_{t=1}^{k} Y_{t}\right\|>2^{(2 / p)} \varepsilon\left(2^{l-1}\right)^{(1 / p)}\right) .
\end{aligned}
$$

By Borel-Cantelli lemma, the formula above implies that, as $l \longrightarrow \infty$, with probability 1 ,

$$
\frac{1}{\left(2^{l-1}\right)^{(1 / p)}} \max _{1 \leq k \leq 2^{l}}\left\|\sum_{t=1}^{k} Y_{t}\right\| \longrightarrow 0 \text {. }
$$

Meanwhile, for any fixed $n$, there exists positive integer $l$ such that $2^{l-1} \leq n<2^{l}$. Hence, with probability 1 , one has

$$
n^{-(1 / p)}\left\|\sum_{t=1}^{k} Y_{t}\right\| \leq \frac{1}{\left(2^{l-1}\right)^{(1 / p)}} \max _{1 \leq k \leq 2^{l}}\left\|\sum_{t=1}^{k} Y_{t}\right\| \longrightarrow 0 .
$$

\section{Conclusion}

In this study, the concept of $m$-CNA random vectors is introduced as a natural extension of CNA random vectors. The weak law of large numbers, complete convergence, and complete moment convergence for linear process of $\mathrm{H}$-valued $m$-CNA random vectors with random coefficients are established. As a corollary of the complete convergence, the strong law of large numbers is also obtained. These results improve and generalise the corresponding ones of recent works such as Hien and Thanh [9], Huan et al. [18], Huan [20], Ko [21], and Ko [23].

However, there are still two open problems should be conquered. In specific, the Baum-Katz type theorem is only extended under the restriction $1 \leq p<2$; the first problem is that whether it is possible to release to $p \geq 2$ ? Another problem is whether the moment condition $\sum_{j \in \mathscr{B}} E\left|X^{(j)}\right| \ln \left(1+\left|X^{(j)}\right|\right)<\infty$ for the strong law of large numbers with $p=1$ can be weakened to $\sum_{j \in \mathscr{B}} E\left|X^{(j)}\right|<\infty$ ?.

\section{Data Availability}

No data were used to support the findings of the study.

\section{Conflicts of Interest}

The author declares no conflicts of interest.

\section{Acknowledgments}

This work was supported by the Key Research Projects of Natural Science in Colleges and Universities of Anhui (KJ2020A0908) and the Key Research Projects of Natural Science of Wuhu Institute of Technology (Wzyzrzd202006).

\section{References}

[1] K. Joag-Dev and F. Proschan, "Negative association of random variables with applications," The Annals of Statistics, vol. 11, no. 1, pp. 286-295, 1983.

[2] T. K. Chandra and S. Ghosal, "Extensions of the strong law of large numbers of Marcinkiewicz and Zygmund for dependent variables," Acta Mathematica Hungarica, vol. 71, no. 4, pp. 327-336, 1996.

[3] T. C. Hu, C. Y. Chiang, and R. L. Taylor, "On complete convergence for arrays of rowwise $m$-negatively associated random variables," Nonlinear Analysis: Theory, Methods and Applications, vol. 71, no. 12, pp. 1075-1081, 2009.

[4] L. X. Zhang and X. Y. Wang, "Convergence rates in the strong laws of asymptotically negatively associated random fields," Applied Mathematics-A Journal of China Universities, Series B, vol. 14, no. 4, pp. 406-416, 1999.

[5] L. X. Zhang, "Strassen's law of the iterated logarithm for negatively associated random vectors," Stochastic Processes and Their Applications, vol. 95, no. 2, pp. 311-328, 2001.

[6] M. H. Ko, T. S. Kim, and K. H. Han, "A note on the almost sure convergence for dependent random variables in a Hilbert space," Journal of Theoretical Probability, vol. 22, pp. 506-513, 2009.

[7] Y. Miao, "Hajeck-renyi inequality for dependent random variables in Hilbert space and applications," Revista De La Unión Matemática Argentina, vol. 53, no. 1, pp. 101-112, 2012.

[8] L. V. Thanh, "On the almost sure convergence for dependent random vectors in Hilbert spaces," Acta Mathematica Hungarica, vol. 139, no. 3, pp. 276-285, 2013.

[9] N. T. T. Hien and L. V. Thanh, "On the weak laws of large numbers for sums of negatively associated random vectors in Hilbert spaces," Statistics and Probability Letters, vol. 107, pp. 236-245, 2015.

[10] J. T. Hu, G. X. Sui, X. X. Lv, and X. D. Li, "Fixed-time control of delayed neural networks with impulsive perturbations," Nonlinear Analysis: Modelling and Control, vol. 23, no. 6, pp. 904-920, 2018.

[11] X. D. Li, J. H. Shen, and R. Rakkiyappan, "Persistent impulsive effects on stability of functional differential equations with finite or infinite delay," Applied Mathematics and Computation, vol. 329, pp. 14-22, 2018.

[12] D. Yang, X. D. Li, J. H. Shen, and Z. J. Zhou, "State-dependent switching control of delayed switched systems with stable and unstable modes," Mathematical Methods in the Applied Sciences, vol. 41, no. 16, pp. 6968-6983, 2018.

[13] D. Yang, X. D. Li, and J. L. Qiu, "Output tracking control of delayed switched systems via state-dependent switching and dynamic output feedback," Nonlinear Analysis: Hybrid Systems, vol. 32, pp. 294-305, 2019.

[14] L. D. Fang, S. H. Ding, H. P. Ju, and L. Ma, “Adaptive fuzzy control for nontriangular stochastic high-order nonlinear 
systems subject to asymmetric output constraints," IEEE Transactions on Cybernetic, 2020.

[15] L. D. Fang, S. H. Ding, H. P. Ju, and L. Ma, "Adaptive fuzzy control for stochastic high-order nonlinear systems with output constraints," IEEE Transactions on Fuzzy Systems, 2020.

[16] K. Q. Mei, L. Ma, Q. X. He, and S. H. Ding, "Finite-time controller design of multiple integrator nonlinear systems with input saturation," Applied Mathematics and Computation, vol. 372, Article ID 124986, 2020.

[17] J. H. Yuan, S. H. Ding, and K. Q. Mei, "Fixed-time SOSM controller design with output constraint," Nonlinear Dynamics, vol. 102, no. 3, pp. 1567-1583, 2020.

[18] N. V. Huan, N. V. Quang, and N. T. Thuan, "Baum-Katz type theorems for coordinatewise negatively associated random vectors in Hilbert spaces," Acta Mathematica Hungarica, vol. 144, no. 1, pp. 132-149, 2014.

[19] L. E. Baum and M. Katz, "Convergence rates in the law of large numbers," Transactions of the American Mathematical Society, vol. 120, pp. 108-123, 1965.

[20] N. V. Huan, "On complete convergence for sequences of random vectors in Hilbert spaces," Acta Mathematica Hungarica, vol. 147, no. 1, pp. 205-219, 2014.

[21] M. H. Ko, "The complete moment convergence for CNA random vectors in Hilbert spaces," Journal of Inequalities and Applications, vol. 2017, p. 290, 2017.

[22] X. Huang and Y. F. Wu, "Strong convergence theorems for coordinatewise negatively associated random vectors in Hilbert space," Journal of Inequalities and Applications, vol. 2018, p. 86, 2018.

[23] M. H. Ko, "On complete moment convergence for CAANA random vectors in Hilbert spaces," Statistics and Probability Letters, vol. 138, pp. 104-110, 2018.

[24] A. Shen, Y. Zhang, B. Xiao, and A. Volodin, "Moment inequalities for $m$-negatively associated random variables and their applications," Statistical Papers, vol. 58, no. 3, pp. 911-928, 2017.

[25] A. Kuczmaszewska, "On complete convergence in Marcinkiewicz-Zygmund type SLLN for negatively associated random variables," Acta Mathematica Hungarica, vol. 128, pp. 116-130, 2010.

[26] S. H. Sung, "Moment inequalities and complete moment convergence," Journal of Inequalities and Applications, vol. 2009, Article ID 271265, 2009. 